\title{
Factores psicoeducativos que determinan la propensión al accidente deportivo en escolares: Diseño y validación de un cuestionario de evaluación
}

\author{
Pedro A. Latorre-Román ${ }^{1 *}$, José C. Cámara-Pérez ${ }^{2}$, Antonio Pantoja-Vallejo ${ }^{3}$ y Tomás Izquierdo-Rus ${ }^{4}$ \\ 1 Departamento de Didáctica de la Expresión Musical, Plástica y Corporal. Universidad de Jaén \\ 2 Junta de Andalucía \\ 3 Departamento de Métodos de Investigación y Diagnóstico en Educación. Universidad de Jaén \\ 4 Departamento de Métodos de Investigación y Diagnóstico en Educación. Universidad de Murcia
}

\begin{abstract}
Resumen: El propósito de este estudio ha sido el diseño y validación de una escala de propensión al accidente deportivo en escolares. La muestra ha estado formada por 254 niños $(M=14.20, D T=1.3)$. Las propiedades psicométricas de la escala se obtuvieron mediante un proceso de validez de contenido y un análisis factorial de los componentes principales. Como resultados obtuvimos un cuestionario de 27 ítems formado por cinco factores y ajustado a los tópicos de validez y fiabilidad científica. Se encontraron diferencias en todos los factores de la escala entre niños y niñas y sólo en el factor búsqueda de sensaciones al considerar la edad de los sujetos. Como conclusión, esta escala es un instrumento inédito que permite la organización de los recursos materiales y didácticos en el deporte de acuerdo a aspectos psicoeducativos relacionados con la propensión al accidente de los niños, lo que es esencial en la prevención de lesiones.

Palabras clave: Factores psicoeducativos; escolares; accidente deportivo; validación.
\end{abstract}

Title: Psychoeducational factors that determine the propensity to school sports accidents: Design and validation of an assessment questionnaire

Abstract: The purpose of this study has been the design and validation of a propensity scale of sports injuries in schools. The sample for the study has been formed of 254 children $(M=14.20, D T=1.3)$. Psychometric properties of the scale were obtained through a process of content validity and a factorial analysis of main components. As a result, we obtained a questionnaire of 27 items consisting of 5 factors and adjusted to validity topics and scientific reliability. Significant differences were found in all factors of the scale in relation to sex, whilst significant differences were only observed in relation to the sensation seeking factor when considering the age of the subjects. This scale is an essential tool for preventing accidents and injuries in school sport by allowing the identification of sports accident-prone students.

Key words: Psychoeducational factors; children; sports accident; validation.

\section{Introducción}

La práctica físico-deportiva conlleva indudables beneficios para la salud, sin embargo, supone en algunos casos un elevado riesgo de lesiones y accidentes. Mientras los gobiernos de los países desarrollados siguen promocionándola entre los niños, hay que tener en cuenta el riesgo de accidentes y la falta de medidas preventivas eficaces en relación con los diferentes factores internos que determinan la seguridad en el deporte (Latorre y Muñoz, 2011). Como consecuencia de la importancia que este problema representa en el contexto deportivo, diversos estudios se han orientado hacia la identificación y el control de los diferentes factores que pueden incidir en el riesgo de los deportistas a lesionarse. Concretamente, determinados aspectos psicológicos y de la personalidad pueden establecer que un sujeto sea más propenso a adoptar conductas de riesgo y, por lo tanto, a accidentarse y lesionarse.

Existe numerosa bibliografía que define a las personas con facilidad para la producción de lesiones como «personalidad propensa a la lesión», y les atribuye una serie de características como agresividad, cólera, capacidad de atención, tendencia a sentirse ofendido y en general, estado de ansiedad permanente (Pascual y Aragües, 1998). En todo caso, los factores psicológicos o relacionados con la personalidad que más se han estudiado al respecto, se asocian con el estrés, la ansiedad competitiva, la impulsividad, las motivaciones de

* Dirección para correspondencia [Correspondence address]:

Pedro Á. Latorre-Román. Departamento de Didáctica de la Expresión Musical, Plástica y Corporal. Universidad de Jaén, Paraje de las Lagunillas s/n, Edificio D2, Despacho 142. 23071, Jaén (España). E-mail: platorre@,ujaen.es logro, el locus control, la búsqueda de sensaciones, la competencia percibida y autoconfianza, el autocontrol y los recursos de afrontamiento y la percepción del riesgo (Abenza, Olmedilla, Ortega y Esparza, 2009; Andersen y Williams, 1999; Greening, Stoppelbein, Chandler y Elkin 2005; Hansen, 2005; Ivarsson y Urban, 2010; Jack y Ronan 1998; Kontos, 2004; Maddison y Prapavessis, 2007; Morrongiello y Rennie, 1998; Zuckerman, 1979).

A nivel infantil, otras características individuales como la edad, el sexo, la experiencia y el temperamento pueden influir en la toma de decisiones de los niños y por lo tanto en la participación en actividades de riesgo (Morrongiello y Lasenby-Lessard, 2007). La asunción de riesgos en el juego permite a los niños aprender la evaluación de riesgos, desarrollar un sentido del riesgo y la comprensión de las características individuales que pueden contribuir a la lesión (Little y Wyver, 2010).

En un análisis de diversos autores, López y Osca (2007) indican que los jóvenes se caracterizan por percibir niveles relativamente bajos de riesgo, subestiman la posibilidad de determinados riesgos, no los perciben de una forma holística, los detectan más lentamente y tienden a sobreestimar sus destrezas. Kontos (2004) destaca que altos niveles de toma de riesgos, bajos niveles de riesgo percibido y bajos niveles de estimación de habilidad, son factores de riesgo de lesiones. Además, las niñas reportan mayores niveles de riesgo percibido y niveles más bajos de asunción de riesgos que los varones. Por tanto, las niñas muestran mayor temor y menos euforia en respuesta a los peligros que los niños (Peterson, Brazeal, Oliver, y Bull (1997). En este sentido, Hillier y Gorronguiello (1998) comprobaron que los niños valoraban varios grados de riesgo, entre ellos, valoraban más bajo el riesgo que las chicas, y que los escolares de 6 años de edad ob- 
servaban menos riesgos y más lentamente que los de 10 años. Por su parte, Morrongiello (1997) y Schwebel y Barton (2005) indican que los niños, con más frecuencia atribuyen sus lesiones a la mala suerte, lo que les lleva a experimentar lesiones repetitivas, mientras que las niñas atribuyen las lesiones a sus propios comportamientos, haciendo que alteren su conducta en situaciones futuras para evitar lesiones. Por tanto, los niños participan en actividades de mayor riesgo físico que las niñas (Morrongiello y Rennie, 1998). Además, socialmente, los chicos perciben menos riesgo en los deportes, de acuerdo con el estereotipo masculino imperante para éstos. En cambio, para las niñas, su estereotipo deportivo está más alejado de la agresividad, contacto físico y la asunción de riesgos que se consideran necesarias en los deportes de contacto como el fútbol. Por tanto, desde un punto de vista psicosocial, a las niñas se les enseña a desconfiar de los riesgos y a los niños a aceptarlos (Harris y Miller, 2000). La falta de experiencia y no preocuparse por las consecuencias graves, pueden desensibilizar a los niños sobre los riesgos potenciales para su salud (Greening et al. 2005). En este sentido, Horvath y Zuckerman (1993) han sugerido que las experiencias pasadas exitosas con la asunción de riesgos puede llevar a una reducción del riesgo percibido, aumentando potencialmente la posibilidad de que un deportista corra riesgos en el deporte.

La auto-eficacia y la competencia percibida podrían influir también en el riesgo percibido y en la asunción de los mismos. Bandura (1997) sugiere que los atletas que presentan una alta autoeficacia son más propensos a intentar habilidades difíciles, por lo tanto, deben ser más propensos a participar en la toma de riesgos en el deporte, así, algunos individuos pueden, de hecho, sobrestimar su capacidad o se perciben a sí mismos a ser mejores de lo que realmente son. Una sobreestimación de la capacidad puede dar lugar a una sensación de invencibilidad y a la decisión de involucrarse en conductas de mayor riesgo. En este sentido, Kontos (2000) en un estudio realizado con una muestra de 253 futbolistas (chicos y chicas, entre 11 y 15 años), mostró que la sobreestimación de la capacidad se relacionaba positivamente con la asunción de riesgos, todo ello, unido a una inexacta baja percepción de riesgo de lesiones, puede llevar a un deportista a ser imprudente en el deporte y a aumentar su probabilidad de lesionarse.

La ansiedad competitiva puede ser un factor de predisposición a la lesión, así Olmedilla, Andreu, Ortín y Blas (2009) destacan la importancia de la ansiedad en la aparición de lesiones. En un estudio con futbolistas, Abenza, Olmedilla y Ortega (2010) señalan que los deportistas con menores niveles de autoconfianza y mayor ansiedad sufren lesiones graves y muy graves. En todo caso, los estudios que relacionan tanto la ansiedad y la autoconfianza con la propensión a la lesión han dado resultados contradictorios.

La búsqueda de sensaciones, dentro de los factores de la personalidad, ha sido uno de los elementos de estudio más analizado en su relación con las conductas de riesgo y en este caso con la accidentalidad y la percepción del riesgo. Zuc- kerman (1979) considera que la búsqueda de sensaciones supone una necesidad de experimentar variadas y complejas sensaciones y el deseo de correr riesgos físicos y sociales por el simple deseo de disfrutar de tales experiencias. El alto buscador de sensaciones se caracteriza por su tendencia a hacer aquellas cosas que un bajo buscador de sensaciones consideraría peligrosas y arriesgadas, la diferencia entre unos y otros es la distinta valoración que se hace del riesgo. Los buscadores de sensaciones asumen varios tipos de riesgos, entre ellos, asumen riesgos físicos en deportes peligrosos (Zuckerman, 1983). Las diferencias sexuales en el rasgo de búsqueda de sensaciones parece que se pueden deber tanto a factores biológicos como a factores de socialización; en cuanto a la edad parece existir una relación negativa entre edad y búsqueda de sensaciones (Chico, 2000).

Los trabajos sobre evaluación de las conductas de riesgo de los niños en situaciones deportivas resultan escasos, la mayoría de investigaciones sobre la toma de decisiones en relación con el riesgo han utilizado las tareas de conveniencia, que exigen que el niño se imagine a sí mismo en diferentes situaciones, con poca relación a situaciones de la vida real que suponen un riesgo de daño físico. Una de las críticas realizadas a este enfoque es la de haber trabajado con listas de riesgos seleccionadas de una forma bastante arbitraria (Cutter, 1993; Hendrickx, 1991) que incluyen riesgos representativos de ámbitos muy dispares. Por tanto, la selección de tareas es un aspecto importante a la hora de estudiar los riesgos (Morrongiello y Rennie, 1998). Es importante destacar la escala de propensión al riesgo de Meertens y Lion (2008) que representa una medida sencilla de 7 ítems muy genéricos, que valora la tendencia individual a exponerse al riesgo. En la evaluación de la percepción del riesgo en el deporte, quisiéramos destacar los estudios de Siesmaa, Blitvich, White y Finch (2011) y Turnock, Finch y Blitvich, (2009) en los que se desarrolla una encuesta para la medición de la participación de los niños en el deporte, la percepción del riesgo y la historia de lesiones. De manera más específica, y centrados en el contexto escolar, Latorre y Pantoja (2012) han desarrollado un cuestionario de percepción del riesgo basándose en la valoración de éste mediante diferentes reactivos (imágenes), que representan tareas deportivas propias del entorno escolar, materiales e instalaciones deportivas.

La forma en que los sujetos se comportan en sus actividades deportivas en relación con la precisión a la hora de percibir, identificar y asumir los riesgos, de disponer de recursos de afrontamiento adecuados a la competición y la competencia motriz necesaria para desenvolverse eficazmente y de manera segura en el deporte; representan elementos muy interesantes en el estudio de la accidentalidad deportiva, sobre todo, en población escolar. Lo que hace necesario la existencia de instrumentos que permitan identificar el valor de aquellos factores que pueden predisponer a un sujeto al accidente deportivo. La escasez de este tipo de instrumentos, específicos para el deporte escolar, motivó la creación de la escala de propensión al accidente deportivo (EPAD). 
El objetivo principal de este estudio es desarrollar un instrumento que nos permita identificar a los escolares que presentan mayor propensión al accidente deportivo basándonos en determinados factores psicológicos y de personalidad. De manera específica pretendemos describir las diferencias por sexo y edad en cuanto a los factores que determinan la propensión al accidente deportivo en escolares de Educación Secundaria.

\section{Método}

\section{Participantes}

El análisis psicométrico de la escala se llevó a cabo mediante el estudio de las respuestas a los reactivos de la misma por parte de la muestra productora de datos, constituida por alumnado de primer y segundo ciclo de Educación Secundaria de un centro público de la provincia de Jaén (España). La muestra tiene por tanto un carácter incidental y no probabilístico, siendo de un total de 254 niños, de entre 12 a 17 años $(M=14.20, D T=1.3)$, de los cuales, 115 son niños $\mathrm{y}$ 139 niñas. El centro escolar se sitúa en una localidad de menos de 5.000 habitantes y eminentemente agrícola.

\section{Instrumento}

La escala EPAD, es un cuestionario de autoinforme compuesto inicialmente por 36 ítems que describen unas variables que condicionan la posibilidad de sufrir un accidente deportivo. En el protocolo del instrumento se solicita que se cumplimenten las cuestiones expresadas en éste de acuerdo a una escala tipo Likert (1, significa muy en desacuerdo y 6 , muy de acuerdo). El diseño y elaboración de la escala se realizó en tres fases. En una primera, se realizó una revisión bibliográfica de aquellos factores relacionados con el propio sujeto que condicionaban la accidentalidad en el deporte, destacándose elementos como la competencia percibida, la búsqueda de sensaciones y la percepción del riesgo. En este proceso se destaca la escasez de instrumentos específicos que analizan esta circunstancia en el ámbito deportivo escolar; existiendo sólo referencias indirectas a las conductas de riesgo de los adolescentes basadas en la escala de búsqueda de sensaciones para niños y adolescentes (Pérez, Ortet, Plá, y Simó, 1987), derivada de la "Escala de Búsqueda de Sensaciones" (Sensation Seeking Scale, SSS, Zuckerman, Eysenck y Eysenck, 1978); y a la percepción del riesgo de Latorre y Pantoja (2012). Teniendo en cuenta estos antecedentes se empezaron a elaborar una serie de cuestiones referenciadas a los factores psicológicos y de personalidad que determinan la accidentalidad, ampliamente descritos en la literatura y adaptadas al ámbito de la actividad físico-deportiva escolar. Se emplearon como referencias, variables de la escala de búsqueda de sensaciones de Pérez et al. (1987) y del cuestionario de autoconcepto físico "CAF" de Goñi, Ruiz de Azúa, y Liberal (2004). En una segunda fase, y como elemento de validez de contenido, se seleccionó a un grupo de seis expertos de Educación Física, con experiencia investigadora en el ámbito de la accidentalidad deportiva. Se les solicitó un juicio crítico sobre los elementos iniciales de la escala, lo cual condujo a la eliminación de 2 ítems basándonos en su contenido, inteligibilidad, y en los que los jueces presentaban discrepancias en la valoración (prueba de concordancia de Kendall). En una tercera fase, se procedió a la validación de constructo del instrumento con una muestra de 254 alumnos.

\section{Procedimiento}

Previamente a la administración de los cuestionarios se solicitó el permiso de la dirección del centro y la colaboración del profesorado de Educación Física. Asimismo, se informó al alumnado del grado de confidencialidad de los cuestionarios, así como del carácter voluntario de su participación. De manera auto-administrada se cumplimentó el cuestionario por pequeños grupos de clase (20 alumnos) en presencia de un investigador del estudio, atendiéndose en todo momento las dudas surgidas y garantizándose la confidencialidad y el anonimato de las respuestas. La duración de aplicación del cuestionario fue de aproximadamente $30 \mathrm{mi}$ nutos. A su vez, se contabilizaron el número de lesiones sufridas por el alumnado en sus actividades deportivas escolares y extraescolares a lo largo de cuatro meses. La recogida de datos se realizó en la primera semana del mes de enero del 2011. El estudio fue aprobado por el Comité de Ética de la Universidad de Jaén.

Los datos de este estudio se han analizado mediante el programa estadístico SPSS., v.18.0 para Windows, (SPSS Inc, Chicago, USA). Para el análisis de datos se ha utilizado la estadística descriptiva (medias, DT) análisis factorial (Varimax con Kaiser), correlaciones (Pearson y CCI) y diferencias de medias (MANOVA) a un nivel de significación de $p<.05$.

\section{Resultados}

Una vez confirmado que el tamaño muestral era adecuado para estudiar la calidad técnica del instrumento, pues se cumple holgadamente el supuesto básico de que si se va a hacer un análisis factorial, la muestra no debe bajar de 150 ó 200 sujetos (Morales, Urosa y Blanco, 2003) o que el número de sujetos recomendado debe ser entre dos y diez veces el número de ítems (Pallás y Villa, 2004), se analizaron las evidencias de validez de la escala. El objetivo principal es comprobar si los 34 ítems de la escala se pueden resumir de alguna forma, es decir, si existen rasgos comunes entre ellos. Paso previo al análisis de la validez de constructo se calculó la homogeneidad del cuestionario, lo que nos permitió eliminar 7 ítems que presentaban un bajo nivel de discriminación y por tanto una correlación $<200$ con el total de la escala, de acuerdo con las recomendaciones de Elbel (1965). Quedándose constituida la escala con 27 ítems. Se recurre al análisis factorial por el método de los componentes principales, como forma de agrupar estos reactivos en factores o componentes que puedan explicar la varianza observada en las respuestas ofrecidas por los sujetos (Escalante y Caro, 
2006). Seguidamente, analizamos el grado de correlación entre las variables, que ha de ser alto para poder realizar el análisis factorial. El índice de adecuación muestral KMO alcanza un valor de .872 y la prueba de esfericidad de Bartlett llega a $2402.003(p<.001)$. Estos datos hacen que se rechace la hipótesis nula de que la matriz de correlación inter-ítems es identidad y se considera que las respuestas están sustancialmente relacionadas, lo que justifica la realización del análisis factorial.

A continuación, determinamos las comunalidades o proporción de varianza que es explicada por los factores comunes. En general, al no existir valores próximos a cero, se puede afirmar que los 27 ítems son explicados por los componentes. El análisis de los componentes principales y Rotación Varimax puso de manifiesto, tras ocho iteraciones, la convergencia en cinco factores que explican un $52.260 \%$ de la varianza. La primera componente es la que más cantidad de varianza explica $(26.839 \%)$, decreciendo hasta llegar a la última componente que sólo lo hace en un 4.6\%. Los ítems quedaron configurados de la forma que se puede apreciar en la Tabla 1 ordenados de acuerdo al grado de saturación. Todos los ítems, excepto el ítem 11, presentan una carga factorial superior a 0.3 . Hemos decidido conservar este ítem ya que presenta una adecuada correlación con el total de la escala y por aportar información importante a la descripción del factor 4.

1. Factor 1. Competencia percibida.

2. Factor 2. Búsqueda de sensaciones.

3. Factor 3. Competitividad.

4. Factor 4. Asunción de riesgos.

5. Factor 5. Percepción del riesgo.

Tabla 1. Matriz de componentes rotados y coeficiente alfa Cronbach por factor.

\begin{tabular}{|c|c|c|c|c|c|}
\hline Items & $\begin{array}{c}\text { Factor } \\
1 \\
\end{array}$ & $\begin{array}{c}\text { Factor } \\
2\end{array}$ & $\begin{array}{c}\text { Factor } \\
3\end{array}$ & $\begin{array}{c}\text { Factor } \\
4\end{array}$ & $\begin{array}{c}\text { Factor } \\
5 \\
\end{array}$ \\
\hline 1. Tengo unas excelentes cualidades físicas. & .814 & & & & \\
\hline 26. Tengo más habilidad que la gente de mi edad practicando deportes. & .777 & & & & \\
\hline 27. Soy capaz de hacer ejercicios y habilidades muy complicadas. & .761 & & & & \\
\hline 2. Tengo mucha confianza en mis capacidades físicas. & .754 & & & & \\
\hline 25. Siempre soy de los mejores en los deportes. & .748 & & & & \\
\hline 13. Tengo capacidades y poderío físico para realizar cualquier actividad deportiva. & .738 & & & & \\
\hline 14. Tengo mucha energía física. & .64 & & & & \\
\hline 9. Me siento muy orgulloso de lo que soy y de lo que puedo hacer físicamente. & .518 & & & & \\
\hline 24. Bucearía en grandes profundidades marinas. & & .754 & & & \\
\hline 7. Me gusta tirarme desde el trampolín más alto de la piscina. & & .613 & & & \\
\hline 6. Me resulta interesante cruzar a nado un río. & & .586 & & & \\
\hline 18. Me gusta montarme en una montaña rusa. & & .569 & & & \\
\hline 5. Siempre soy el primero en ofrecerme para hacer las actividades más arriesgadas. & & .557 & & & \\
\hline 2. Algunas veces me gusta hacer cosas que impliquen pequeños sobresaltos. & & .54 & & & \\
\hline 23. Me encantan los grandes toboganes de los parques acuáticos. & & .476 & & & \\
\hline 3. Necesito superarme para ser el mejor. & & & 699 & & \\
\hline 15. Cuando pierdo me siento muy mal conmigo mismo. & & & 687 & & \\
\hline 4. Me gusta siempre ser el mejor y sorprender a los demás. & & & .594 & & \\
\hline 16. Me gusta ser el capitán del equipo. & & & .463 & & \\
\hline 17. Suelo soltarme de manos al conducir la bicicleta. & & & & .739 & \\
\hline 19. Conduzco la bicicleta sin casco. & & & & .74 & \\
\hline 8. Soy capaz de tirarme de cabeza desde una roca al mar. & & & & .398 & \\
\hline 11. Hago deporte estando lesionado. & & & & .251 & \\
\hline 21. Desplazarse haciendo el equilibrio invertido (el pino) no es peligroso para mí. & & & & & .698 \\
\hline 12. Hacer una voltereta en el aire es poco peligroso. & & & & & .684 \\
\hline 1. Como sé mucho nadar, hacer piragüismo sin chaleco salvavidas no supone ningún riesgo para mí. & & & & & .631 \\
\hline 22. Saltar una valla de atletismo no conlleva ningún peligro. & & & & & .529 \\
\hline Coeficientes Alfa de Cronbach & .889 & .752 & 672 & 621 & .643 \\
\hline
\end{tabular}

En todos los factores, excepto el factor 5 sobre percepción del riesgo, a mayor puntuación mayor valoración de la escala, sin embargo, en el factor 5 a mayor puntuación, menor percepción del riesgo. Una vez definida la validez de la escala, calculamos la fiabilidad de la misma mediante el coeficiente alfa de Cronbach, que alcanza un valor de .879 , lo que indica una alta consistencia interna del instrumento, o lo que es lo mismo, que es confiable. En todos los valores se observa que la relación de cada ítem con el total mantiene valores muy estables, en todos los casos por encima de .87 .
Para completar este análisis pormenorizado del cuestionario, a una muestra aleatoria de 25 alumnos, se aplicó un retest pasadas 5 semanas. Se obtuvieron coeficientes de correlación intraclase (CCI), para el primer factor de .918, para el segundo factor de .916, para el tercer factor de .726, para el cuarto de .899 y finalmente para el quinto de .791 . Todo ello, nos indica que el cuestionario presenta una adecuada estabilidad en el tiempo.

Por último, como análisis de validez concurrente se calculó la correlación Pearson de las dimensiones del cuestiona- 
rio EPAD con la puntuación total de la escala de búsqueda de sensaciones para niños y adolescentes (EBS-J) de Pérez et al. (1987). Podemos observar una correlación muy significativa entre todas las dimensiones del EPAD y la puntuación total de la escala EBS-J (Tabla 2).

En la Tabla 2 se exponen las correlaciones entre los diferentes factores de la escala EPAD, con el número de lesio- nes y accidentes recogidos en los alumnos a lo largo de cuatro meses y la puntuación total de la escala EBS-J. Podemos observar que el número de lesiones se correlaciona significativamente con la competencia percibida, la competitividad y la asunción de riesgos. A su vez, todos los factores de la escala EPAD presentan correlaciones significativas entre sí y con la escala EBS-J.

Tabla 2. Correlaciones entre los diferentes factores de la escala EPAD, número de lesiones y accidentes y el total de la escala EBS-J.

\begin{tabular}{|c|c|c|c|c|c|c|c|}
\hline & $\begin{array}{c}\text { Número } \\
\text { de lesiones }\end{array}$ & $\begin{array}{c}\text { Competencia } \\
\text { percibida }\end{array}$ & Competitividad & $\begin{array}{l}\text { Asunción } \\
\text { de riesgos }\end{array}$ & $\begin{array}{c}\text { Búsqueda de } \\
\text { sensaciones }\end{array}$ & $\begin{array}{c}\text { Percepción } \\
\text { del riesgo }\end{array}$ & $\begin{array}{c}\text { EBS-J } \\
\text { Total escala }\end{array}$ \\
\hline Número de lesiones & 1 & $.231(* *)$ & $.185(* *)$ & $.266(* *)$ & .15 & .7 & .116 \\
\hline Competencia percibida & & 1 & $.477(* *)$ & $.353(* *)$ & $.41(* *)$ & $.299(* *)$ & $.232(* *)$ \\
\hline Competitividad & & & 1 & $.368(* *)$ & $.31(* *)$ & $.24(* *)$ & $.27(* *)$ \\
\hline Asunción de riesgos & & & & 1 & $.472(* *)$ & $.433(* *)$ & $.462(* *)$ \\
\hline Búsqueda de sensaciones & & & & & 1 & $.287(* *)$ & $.629(* *)$ \\
\hline Percepción del riesgo & & & & & & 1 & $.31(* *)$ \\
\hline
\end{tabular}
$* * p<.01$

En relación con los resultados obtenidos en la muestra, tras un análisis de varianza multivariado (MANOVA), considerando como variables de respuesta las diferentes dimensiones de la escala EPAD y el número de lesiones y como factores explicativos la edad y el sexo, se puede observar en la tabla 3 como los varones superan en puntuación a las mu- jeres en todas las dimensiones de la escala EPAD y en el número de lesiones. En la tabla 4, en relación con la edad, se comprueba que sólo en la dimensión de búsqueda de sensaciones hay diferencias por edad, incrementándose en los niños mayores.

Tabla 3. Medias y desviaciones típicas de las subescalas EPAD, número de lesiones y diferencias según el sexo.

\begin{tabular}{lccccc}
\hline Sexo & Niños $(n=115)$ & Niñas $(n=139)$ & $F$ & $p$ & Eta cuadrado \\
Competencia percibida & $M(D T)$ & $M(D T)$ & Potencia & \\
observada & & & \\
Competitividad & $34.26(7.65)$ & $25.33(7.93)$ & 80.645 & .000 & .244 \\
Asunción de riesgos & $16.56(4.43)$ & $11.86(4.56)$ & 80.645 & .000 & .244 \\
Búsqueda de sensaciones & $15.27(4.58)$ & $10.64(4.84)$ & 66.836 & .000 & .211 \\
Percepción del riesgo* & $31.00(7.02)$ & $27.98(8.08)$ & 60.398 & .002 & .000 \\
Número de lesiones & $12.80(4.94)$ & $9.69(4.62)$ & 9.966 & .000 & .000 \\
\hline
\end{tabular}

*A mayor puntuación menor percepción del riesgo.

Tabla 4. Medias y desviaciones típicas de las subescalas EPAD, número de lesiones y diferencias según la edad.

\begin{tabular}{|c|c|c|c|c|c|c|}
\hline Edad & $\begin{array}{c}12 \text { a } 14 \text { años }(n=144) \\
M \quad(D T)\end{array}$ & $\begin{array}{c}15 \text { a } 17 \text { años }(n=110) \\
M(D T)\end{array}$ & $F$ & $p$ & Eta cuadrado & $\begin{array}{l}\text { Potencia } \\
\text { observada }\end{array}$ \\
\hline Competencia percibida & $29.86(9.12)$ & $28.73(8.77)$ & .701 & .403 & .003 & .133 \\
\hline Competitividad & $14.44(5.13)$ & $13.40(4.95)$ & 2.474 & .117 & .010 & .347 \\
\hline Asunción de riesgos & $12.57(5.21)$ & $12.95(5.33)$ & .810 & .369 & .003 & .146 \\
\hline Búsqueda de sensaciones & $28.18(7.80)$ & $30.88(7.45)$ & 8.099 & .005 & .031 & .809 \\
\hline Percepción del riesgo* & $11.18(5.32)$ & $11.00(4.58)$ & .005 & .943 & .000 & .051 \\
\hline Número de lesiones & $.53(.62)$ & $.48 \quad(.57)$ & .368 & .545 & .001 & .093 \\
\hline
\end{tabular}

$*$ A mayor puntuación menor percepción del riesgo.

En el estudio de las diferencias en función de la interacción de las variables edad y sexo, los resultados no indican un efecto estadísticamente significativo de sexo por edad (Lambda de Wilks $\left.=.993, F_{(6.245)}=.285, p=.944\right)$.

\section{Conclusiones y discusión}

El objetivo de este estudio ha sido elaborar un instrumento de propensión al accidente deportivo en escolares como población muy sensible a esta circunstancia. El cuestionario EPAD constituye un instrumento inédito y original que ha mostrado alta fiabilidad y adecuada validez de contenido y constructo. En cuanto a la fiabilidad, la escala ha mostrado buena consistencia interna con un coeficiente alfa igual a .87 y una adecuada estabilidad temporal test-retest. Las saturaciones de cada uno de los ítems con sus respectivos factores son altas, exceptuando el ítem 11, que se mantuvo por su adecuado poder de discriminación. Por otro lado, las correlaciones entre factores indican cierta relación y dependencia entre ellos. Por todo ello, podemos afirmar que los datos confirman la validez del cuestionario, con una estructura de cinco factores. 
En lo que se refiere a las diferencias por sexo, los niños puntúan más que las niñas en todos los factores. En el factor percepción del riesgo los datos están en consonancia con las aportaciones de Hillier y Gorronguiello (1998) y Kontos (2004), según las cuales, las niñas presentan mayores niveles de riesgo percibido, siendo éstas más precisas que los niños en la evaluación de riesgos en el deporte. Sin embargo, DiLillo, Potss y Himes (1998) no encuentran diferencias en la evaluación del riesgo entre niños y niñas. Little y Wyver (2010) indican además que los niños son capaces de identificar las conductas de riesgo de lesiones, sin embargo, la diferenciación de la gravedad de la lesión potencial es menos precisa. Además, en este estudio no hemos encontrado diferencias debido al incremento de edad, aunque Peterson et al. (1997) sugieren que con el aumento de la edad, los niños perciben las situaciones que tienen menos riesgo.

En relación con el factor de asunción y toma de riesgos, los niños obtienen valores significativamente más elevados que las niñas, datos semejantes a los de Kontos (2004) y Morrongiello y Rennie (1998), esto puede llevar a los niños a participar en actividades de mayor riesgo.

En el rasgo de competitividad se obtienen valores más elevados en los varones de acuerdo a los encontrados en otros estudios (Houston et al. 2005; Jones, Neuman, Altmann y Dreschler, 2001; Remor, 2007). No se ha encontrado asociación entre la edad y la competitividad. En la competencia percibida, relacionada con el autoconcepto físico y las variables definidas por Goñi, Ruiz de Azúa y Liberal (2004), se obtienen valores superiores en hombres al igual que los datos obtenidos por estos autores. Estos valores también coinciden con los aportados por Hellín, Moreno y Rodríguez (2006), Moreno y Cervelló (2005), Moreno, Moreno y Cervello, (2009) y los datos de autoeficacia motriz de Hernández et al. (2011). Por el contrario, Kontos (2004) informa que los niños y niñas presentan una similar estimación y sobreestimación de la habilidad. En relación con la variaciones por la edad, la competencia percibida no se vio afectada por ésta, tanto en hombres como en mujeres, sin embargo, Hernández et al. (2011) sí encuentran este efecto de reducción de la autoeficacia motriz a mayor edad, pero sólo en mujeres.

El factor búsqueda de sensaciones es mayor en niños que en niñas y se incrementa con la edad de acuerdo con las consideraciones de Comín, Torrubia y Mor (1998), Butkovic y Bratko (2003) y Reio y Choi (2001). Los niños que mayores valores presentan en búsqueda de sensaciones experimentan a su vez una mayor asunción de riesgos (Morrongiello y Sedore, 2005), circunstancia que se ve reflejada en este estudio, en consonancia con Little y Wyver (2010).

Por último, es de destacar el mayor nivel de accidentalidad y lesiones en varones, resultados que concuerdan con el estudio epidemiológico en España del Instituto Nacional del Consumo (2008), en el que se indica que la accidentalidad infantil es superior en niños en comparación con las niñas, en todas las franjas de edad, y en particular en el área deportiva (incluido los colegios). En este estudio, hemos encontrado una correlación significativa $(p<.01)$ entre la competitividad, asunción de riesgos, competencia percibida y el número de lesiones, en consonancia con Kontos (2004), el cual señala que la estimación de la capacidad se asocia con un aumento significativo en el riesgo de lesiones.

Los análisis psicométricos del presente estudio confirman la validez y fiabilidad de la versión inicial del EPAD adaptado al deporte escolar. En líneas generales, se puede afirmar que la escala puede servir como instrumento válido para ser utilizado en los centros educativos y deportivos a la hora de detectar de forma sencilla la propensión al accidente deportivo de los niños, sirviendo de base a docentes y profesionales del deporte para adecuar el diseño de las actividades físico-deportivas al nivel de auto eficiencia, personalidad y percepción del riesgo del alumnado en cuestión. Todo ello, como señalan DiLillo, Potss y Himes (1998), nos ofrece conocimiento sobre los determinantes de la valoración de riesgo, útil para orientar las intervenciones de todos los niños, especialmente los que corren mayor riesgo de daño físico a causa de las evaluaciones distorsionadas de los riesgos ambientales, lo que se constituye en un elemento importante de prevención de lesiones. Para los niños, la capacidad de hacer juicios de riesgo en relación con sus propias capacidades en desarrollo es especialmente importante a medida que aprenden las habilidades necesarias para funcionar e interactuar con su entorno. El juego al aire libre es un contexto de especial importancia y oportunidad en que los niños desarrollan la capacidad de percibir y evaluar los riesgos a medida que aprenden a evitar lesiones, mientras desarrollan y dominan una amplia gama de habilidades motrices básicas (Little y Wyver, 2010). La provisión de oportunidades para la toma de riesgos en el juego de los niños al aire libre no quiere decir que la seguridad se pase por alto, más bien significa que los profesores y padres tienen que ser conscientes de los peligros y tomar las medidas necesarias para garantizar que el entorno sea seguro (Henninger, 1994). Los niños necesitan aprender a tomar riesgos calculados. Incluso dentro de la prevención de lesiones y el campo de seguridad del patio de recreo, hay un reconocimiento de la importancia de la asunción de riesgos durante el juego (Little y Wyver, 2008).

Como limitaciones del estudio destacamos el escaso tiempo de seguimiento de la accidentalidad deportiva de la muestra analizada, lo cual ha podido influir en la relación de ésta y los diferentes factores de la escala. Como perspectiva de futuro, sería interesante incrementar el registro de lesiones en un estudio longitudinal más prolongado y con una muestra mayor. Coincidimos con Moreno, Cervelló y Martínez (2007) en la necesidad de realizar trabajos de investigación en ámbitos no competitivos. A su vez, se debería ampliar el estudio a otros ámbitos poblacionales con características sociodemográficas diversas (nivel económico, entornos urbanos y rurales). 


\section{Referencias}

Abenza, L., Olmedilla, A. y Ortega, E. (2010). Effect of injuries on psychological variables among under-19 soccer players. Revista Latinoamericana de Psicología, 42 (2), 265-277.

Abenza, L., Olmedilla, A., Ortega, E. y Esparza, F. (2009). Estados de ánimo y adherencia a la rehabilitación de deportistas lesionados. Apunts Medicina de l'Esport, 161, 29-37.

Andersen, M. B,. \& Williams, J. M. (1999). Athletic injury, psychosocial factors, and perceptual changes during stress. Journal of Sports Sciences, 17 (9), 735-741.

Bandura, A. (1997). Self-efficacy: The exercise of control. New York: Freeman.

Butkovic, A,. \& Bratko, D. (2003). Generation and sex differences in sensation seeking: Results of the family study. Perceptual and Motor Skills, 97 (3), 965-970.

Chico, L. (2000). Búsqueda de sensaciones. Psicothema, 12 (2), 229-235.

Comín, E., Torrubia, R. y Sancho, J. (1998). Relación entre personalidad, actitudes y consumo de alcohol, tabaco y ejercicio en escolares. Gaceta Sanitaria, 12 (6), 255-62.

Cutter, S. L. (1993). Living with Risk. Londres: Edward Arnold.

DiLillo, D., Potss, R., \& Himes, S. (1998). Predictors of Children's Risk Appraisals. Journal of Applied Developmental Psychology, 19 (3), 415-427.

Elbel, R. L. (1965). Measuring educational achievement. Englewoods Cliffs, N. J: Prentice-Hall.

Escalante, E. y Caro, A. (2006). Investigación y análisis estadísticos de datos en SPSS. Mendoza: FEEyE.

Goñi, A., Ruiz de Azúa, S. y Liberal, I. (2004). Propiedades psicométricas de un nuevo cuestionario para la medida del autoconcepto físico. Revista de psicología del deporte, 13 (2), 195-21.

Greening, L., Stoppelbein, L., Chandler, C., \& Elkin, D. (2005). Predictors of Children's and Adolescents' Risk Perception. Journal of Pediatric Psychology, 30(5), 425-435.

Hansen, C.P. (2005). Personality characteristics of the accident involved employee. Journal of Business \& Psychology, 2(4), 346-365.

Harris, M. B. y Miller, K. C. (2000). Gender and perceptions of danger. Sex Roles, 43 (11), 843-863.

Hellín, P., Moreno, J. A. y Rodríguez, P. L. (2006). Relación de la competencia motriz percibida con la práctica físico-deportiva. Revista de psicología del deporte, 15 (2), 219-231.

Hendrickx, L. C. (1991). How Versus How Often. The role of Scenario Information and Frequency Information in Risk Judgment and Risky Decision Making. Groningen: Rijksuniversiteit Groningen.

Henniger, M. L. (1994). Planning for outdoor play. Young Children, 49 (4), $10-$ 15.

Hernández, J. L., Velázquez, R., Martínez, M. G., Garoz, I. y Tejero, C. (2011). Escala de Autoeficacia Motriz: propiedades psicométricas y resultados de su aplicación a la población escolar española. Revista de Psicología del deporte, 20 (1), 13-28.

Hillier, L. \& Morrongiello, B. A. (1998). Age and gender differences in school-age children's appraisal of injury risk. Journal of Pediatric Psychology, 23 (4), 229-238.

Horvath, P., \& Zuckerman, M. (1993). Sensation seeking and risk appraisal and risk behavior. Personality y Individual Differences, 14(1), 41-52.

Houston, J. M., Harris, P. B., Moore, R., Brummett, R,. \& Kmetani, H. (2005). Competitiveness among japanese, chinese, and american undergraduate students. Psychological Reports, 97 (1), 205-212.

Instituto Nacional del Consumo. (2008) Programa de prevención de lesiones: red de detección de accidentes domésticos y de ocio. Madrid: Ministerio de Sanidad, Política Social e Igualdad.

Ivarsson, A,. \& Urban, J. (2010). Psychological factors as predictors of injuries among senior soccer players. A prospective study. Journal of Sports Science and Medicine, 9, 347-352.

Jack, J., \& Ronan K. R. (1998). Sensation seeking among high and low risk sports participants. Personality and Individual Differences, 25 (6), 1063-1083.

Jones, J. W., Neuman, G., Altmann, R., \& Dreschler, B. (2001). Development of the sports performance inventory: A psychological measure of athletic potential. Journal of Business and Psychology, 15 (3), 491-50.

Kontos, A. (2004). Perceived Risk, Risk Taking, Estimation of Ability and Injury Among Adolescent Sport Participants. Journal of Pediatric Psychology, 29 (6), 447-455.
Kontos, A. P. (2000). The effects of perceived risk, risk taking behaviors, and bod size on injury in youth sport. Eugene, OR: Microform Publications, University of Oregon.

Latorre, P. A. y Muñoz. A. (2011). Manual de control de la seguridad de los espacios y equipamientos deportivos de uso escolar. Jaén: Diputación de Jaén.

Latorre, P. y Pantoja, A. (2012). Diseño y validación de una escala de percepción del riesgo en las actividades físico-deportivas escolares. Retos, 21, 25-29.

Little, H, \& Wyver, S. (2008). Outdoor Play: Does Avoiding the Risks Reduce the Benefits? Australian Journal of Early Childhood, 33 (2), 33-40.

Little. H,. \& Wyver, S. (2010). Individual differences in children's risk perception and appraisals in outdoor play environments. International Journal of Early Years Education, 18 (4), 297-313.

López, B. y Osca, A. (2007). Factores explicativos de la accidentalidad en jóvenes: Un análisis de la investigación. Revista de Estudios de Juventud, 79 , 75-89.

Maddison, R, \& Prapavessis, H. (2007). Preventing sport injuries: A case for psychology intervention. In Pargman (Ed.), Psychological bases of sport injuries (pp.25-38). D. Morgantown, WV: Fitness Information Technology.

Meertens, M. R y Lion, R. (2008). Measuring an individual's tendency to take risks: The risk propensity scale. Journal of Applied Social Psychology, 38 (6), 1520.

Morales, P., Urosa, B. y Blanco, A. (2003). Construcción de escalas de actitudes tipo likert. Madrid: La Muralla.

Moreno, J. A. y Cervelló, E. (2005). Physical self-perception in Spanish adolescents: effects of gender and involvement in physical activity. Journal of Human Movement Studies, 48, 291-311.

Moreno, J. A., Cervelló, E. y Martínez (2007). Validación de la escala de medida de motivos para la actividad física-revisada en españoles: Diferencias por motivos de participación. Anales de Psicología, 23 (1), 167-176.

Moreno, J. A., Moreno, R. y Cervelló, E. (2009). Relación del autoconcepto físico con las conductas de consumo de alcohol y tabaco en adolescente. Adicciones, 21 (2), 147-154.

Morrongiello, B. A. (1997). Children's perspectives on injury and close call experiences: Sex differences in injury-outcome processes. Journal of Pediatric Psychology, 22 (4), 499-512.

Morrongiello, B. A,. \& Rennie, H. (1998). Why do boys engage in more risk taking than girls? The role of attributions, beliefs, and risk appraisals. Journal of Pediatric Psychology, 23 (1), 33-43.

Morrongiello, B.A,. \& Sedore, L. (2005). The Influence of Child Attributes and Social-Situational Context on School-Age Children's Risk Taking Behaviors that Can Lead to Injury. Journal of Applied Developmental Psychology: An International Lifespan Journal, 26 (3), 347-361

Morrongiello, B.A,. \& Lasenby-Lessard. J. (2007). Psychological determinants of risk taking by children: An integrative model and implications for interventions. Injury Prevention, 13 (1), 20-25.

Olmedilla, A., Andreu, M. D., Ortín, F. J. y Blas, A. (2009). Ansiedad competitiva y lesiones: factores deportivos y tipos y gravedad de lesión. In ternational Journal of Hispanic Psychology, 2 (2).

Pallás, J.M. y Villa, J. (2004). Métodos de investigación en clínica y epidemiología. Madrid: Elsevier.

Pascual, A. y Aragües, G. (1998). Lesiones deportivas y rasgos de ansiedad en los jugadores de fútbol.Medina Clínica, 111 (2), 45-48.

Pérez, J., Ortet, G., Plá, S. y Simó, S. (1987). Escala de Búsqueda de Sensaciones para Niños y Adolescentes (EBS-J). Evaluación Psicológica, 3 , 283-290.

Peterson, L., Brazeal, T., Oliver, K.. \& Bull, C. (1997). Gender and developmental patterns of affect, belief, and behaviors in simulated injury events. Journal of Applied Developmental Psychology, 18 (4), 531-546.

Reio, T. G.. \& Choi, N. (2004). Novelty seeking in adulthood: Increases accompany decline. Journal of Genetic Psychology, 165 (2), 119-133.

Remor, E. (2007). Propuesta de un cuestionario breve para la evaluación de la competitividad en el ámbito deportivo: competitividad-10. Revisto psicología del deporte, 16 (2), 167-183.

Schwebel, D. y Barton, B. (2005). Contributions of Multiple Risk Factors to Child Injury. Journal of Pediatric Psychology, 30 (7), 553-561. 
Siesmaa, E., Blitvich, J. D., White, P,. \& Finch, C. (2011). Measuring children's self-reported sport participation, risk perception and injury history: Development and validation of a survey instrument. Journal of Science and Medicine in Sport, 14 (1), 22-26.

Turnock, E., Finch, C,. \& Blitvich, J. (2009). Measuring children's sport participation, risk perceptions and injury history: Development and validation of a survey instrument. Journal of Science and Medicine in Sport, 12 (1),
Zuckerman, M. (1979). Sensation Seeking: Beyond the Optimal Level of Arousal. Hillsdale, NY: Erlbaum.

Zuckerman, M. (1983). Sensation seeking and supports. Personality and Individual Differences, 4, 285-292.

Zuckerman, M., Eysenck, S. B,. \& Eysenck, A. J. (1978). Sensation seeking in England and American: cross-cultural age and sex comparisons. Journal of Consulting and Clinical Psychology, 46 (1), 139-149.

(Artículo recibido: 25-04-2012, revisado: 15-06-2012, aceptado: 18-06-2012) 